[Aus dem hygienischen Institut der Universität Breslau.]

\title{
Ueber die freiwillige Eisenausscheidung aus Grundwasser und eine Enteisenungsmethode für Kesselbrunnen.
}

Von

Dr. A. Lübbert,

Docenten der Hygiene in Breslan.

Lässt man eine frisch entnommene Probe Eisenoxydul- und, wie im vorliegenden Falle, auch Sauerstoffhaltigen Grundwassers in einem offenen Glasgefäss an der Luft stehen, so beobachtet man, dass die Eisenausscheidung gewissermaassen in zwei Phasen verläuft. ${ }^{1}$

Je höher die Temperatur, um so früher beginnt die Flüssigkeit in ihrer ganzen Masse gleichmässig gelblichbraun zu opalesciren als Ausdruck einer Ausscheidung von Eisen. Während nun die tieferen Schichten des Wassers vor der Hand unverändert das bald erreichte Maximum jener Opalescenz bewahren, bemerkt man als zweite Phase, dass sich die Oberfläche intensir rothbraun färbot. Flocken ausgeschiedenen Eisenoxydhydrates senken sich zu Boden, die Flüssigkeit wird mehr und mehr getrübt und bald ist eine Differenzirung der anfänglich so auffallenden, von der Oberfläche ausgehenden Ausscheidungszone nicht mehr möglich. Unter Sedimentirung des Fisenoxydhydrates klärt sich die ganze Flüssigkeit und höchstens auf der Oberfläche erhalten sich einige irisirende Häutchen der Eisenverbindung.

1 Es eignen sich für diese Versuche am besten Wässer mit hohem Eisengehalt (20-30 ${ }^{\mathrm{mg}}$ Fe im Liter). 
Jene initiale, die ganze Flüssigkeit schnell durchsetzende, durch Eisenoxydation ${ }^{1}$ bedingte Opalescenz, trat nun schon zu einer Zeit selbst in den tiefsten Schichten des Wassers auf, ehe, der Erfahrung und dem Versuche entsprechend, der Luftsauerstoff in nöthiger Weise absorbirt sein konnte, so dass nur der im Wasser schon ursprünglich vorhandene Sauerstoff auf das gelöste Eisenoxydul gewirkt haben konnte. Hiermit stellt sich aber sofort die Frage, warum jener Sauerstoff nicht schon im Erdboden zur Wirkung kam, oder, was dasselbe bedeutet, wie es möglich ist, das eisenoxydulhaltige Grundwasser freien Sauerstoff enthalten können, und zwar manchmal in solchen Mengen, dass er ausreichen würde, um alles vorhandene Eisenoxydulsalz zu oxydiren. Diese Thatsache, das Vorkommen freien Sauerstoffs neben Eisenoxydulverbindungen, lässt von vornherein vermuthen, dass in dem Grundwasser ein Agens vorhanden sein muss, welches den Sauerstoff verhindern kann, seine oxydirenden Wirkungen auszuüben, und eine Erörterung dieser Frage an der Hand des Experimentes erscheint um so mehr gerechtfertigt, als sich erwarten lässt, dass eine Klarlegung dieser Verhältnisse Enteisenungsmethoden finden lässt, die ohne maschinelle Hülfe, und, was das Wichtigste erscheint, ohne viele eine Infection begünstigende Manipulationen ein Grundwasser nutzbar machen. Entnimmt man Proben Eisenoxydul- und Sauerstoffhaltigen Grundwassers, obue dass Luft mit denselben in Berührung kommt, durch Einsenkung Wasserstoff-gefüllter Flaschen, so beobachtet man zweierlei: Werden die Flaschen unter vollständiger Verdrängung des Wasserstoffs bis an den sicher functionirenden Glasverschluss gefüllt, so kann man diese Proben beliebig lange aufbewahren, ohne dass man je Eisenausscheidung bemerken wird. Füllt man dagegen eine zweite Reihe von Flaschen nur etwa zur Hälfte, so dass man über dem Wasser eine reine Wasserstoffatmosphäre conservirt, so überzeugt man sich, dass Ausfällung von Eisenoxydhydrat eintritt. ${ }^{2}$ Genau dieselben Resultate werden constatirt, wenn an Stelle des Wasserstoffes Stickstoff zur Flaschenfüllung gewählt wurde. War atmosphärische Luft mit dem Wasser nicht in Berührung gekommen, so konnte die Ausscheidung von Eisenoxydhydrat wieder nur dadurch bedingt sein, dass der im Wasser schon ursprünglich vorhandene Sauerstoff wirkte. In den vollständig gefüllten Flaschen nun beobachtete man keine Oxydation, während eine solche in den halbgefüllten Kolben unter der

\footnotetext{
1 Dass es sich schon um Oxydation handelt, ergiebt sich unter Anderem aus dem Verhalten zur Kohlensäure.

${ }^{2}$ Als die Cylinder längere Zeit gestanden hatten, löste sich das ausgefallene Ferrihydrat wieder auf und die Wässer wurden wiederum vollkommen klar. Es ist dies auf die Wirkung von Mikroorganismen zurückzuführen. In einer besonderen Arbeit soll diese interessante Thatsache näher besprochen werden.
} 
Wasserstoff- oder Stickstoffatmosphäre eintrat, so dass wir berechtigt sind, anzunehmen, dass der im. Wasser gelöste Sauerstoff nur dann eben eine Wirkung entfalten kann, wenn ein die Oxydation verhinderndes Gas entweichen kann, wenn also über dem Wasser eine Atmosphäre steht, in welcher der Partialdruck dieses Gases geringer ist als im Wasser selbst.

Wenn unter dem Stickstoff Ausfällung eintrat, so kann dieser selbst natürlich nicht der die Sauerstoffwirkung verhindernde Körper scin, so dass nur noch die Kohlensäure in Betracht käme, für welche ja die Spannung in der Stickstoff- wie Wasserstoffatmosphäre gleich Noll war. Stellen wir nun über das Eisenwasser etwa das doppelte bis dreifache Volumen reiner Kohlensäure, so bleiben die Flaschen dauernd klar als Ausdruck dafür, dass trotz des Vorhandenseins freien Sauerstoffes alles Eisen in Lösung geblieben ist. Bedingung für das Gelingen dieses Versuches ist es freilich, dass der Versehluss der Flasehen nur durch Glas, am besten durch Zuschmelzen bewerkstelligt war. Hätte man Kautschukstopfen gewählt, so bliebe die Beobachtung nicht aus, dass nach einer Reihe von Tagen Eisenausscheidung mit Sicherheit eintritt, da der Kautschuk erstaunlich grosse Mengen Kohlensäure occludirt. Nachdem nun der gut zugeschmolzene Kolben eine Zeit lang beobachtet und klar bleibend befunden worden ist, werde er nunmehr mit seinem ausgezogenen Hals an eine Vorlage angeschlossen, welche mit starker Kalilauge beschickt ist. Wenn man sicher ist, den Versuch so geleitet zu haben, dass jede Spur Sauerstoff aus der Vorlage und ihrer Verbindung mit dem Kolben ferngehalten ist, so bricht man jetzt die im Verbindungsstück steckende, ausgezogene Kolbenhalsspitze ab, damit nach Herstellung der Communication die Kohlensäure des Kolbens von der Kalilauge gebunden werde. Dass natürlich ein Ueberfliessen von Kalilauge aus der Vorlage in den das Eisenwasser haltenden Kolben auf das Sorgfältigste vermieden werden muss, braucht nicht besonders betont zu werden. Wird dieser Versuch des Oefteren wiederholt, so überzeugt man sich, dass der Sauerstoff des Wassers regelmässig schon Eisenoxydhydrat zur Fällung bringt, ehe etwa die gesammte freie Kohlensäure gebunden ist, dass demnach eine zur Wassermasse in Relation stehende bestimmte Menge Kohlensäure nöthig ist, um die 0xydation des gelösten Eisens hintanzuhalten. ${ }^{1}$ Man ist demnach nicht berechtigt zu schliessen, dass die Kohlensäure auf die freiwillige

${ }^{1}$ Aus einer mit Eisenwasser vollständig gefüllten abschliessbaren Flasche kann man natürlich durch Wasserstoff die Kohlensäure bis auf geringste Spuren vertreiben, ohne dass Eisenausfällung stattfinden wird, vorausgesetzt, dass auch eventuell vorhandener Sauerstoff gleichzeitig verjagt war, and zwar schon zu einer Zeit, zu der noch grössere Kohlensäuremengen vorhanden waren. 
Eisenausscheidung ohne Einfluss sei, wenn man auch sieht, dass trotz des Vorhandenseins relativ grosser Mengen freier Kohlensäure Eisenoxydation eintreten kann. Durch die Versuche, welche zeigen, dass sich Eisenoxydul im Wasser neben freiem Sauerstoff nur bei Gegenwart gewisser Mengen Kohlensäure erhalten kann, ist die Bedeutung der Kohlensäure für diesen Sauerstoff klargelegt und es soll im Folgenden gezeigt werden, wie man durch Kohlensäure die Einwirkung einer Atmosphäre von Luft, ja sogar von reinem Sauerstoff beeinflussen kann. Für einen ersten Versuch fülle man drei je $200 \mathrm{cem}$ fassende Bechergläser mit dem Eisenwasser voll an und leite durch das eine reinen Wasserstoff, durch das zweite reine Kohlensäure in gleichmässigem, die Oberfläche nicht aufwirbelndem Strome, während das dritte Becherglas ruhig stehend belassen werde. In der rom Wasserstoff durchperlten Wasserprobe wird die Eisenausscheidung sehr bald am ausgesprochensten sein. Es wird dann, der Intensität der Oxydation gemäss, das ruhig belassene Glas an zweite Stelle zu setzen sein, während unter dem Einfluss der Kohlensäure die Eisenausscheidung noch ganz verhindert sein wird. Der Wasserstoff verdrängt die Kohlensäure aus dem Wasser und veranlasst hiermit eine schnellere Oxydation der Stehprobe gegenüber, während die Durchleitung der Kohlensäure im dritten Glase eine Absorption des Gases bewirkt, die eine Sauerstoffwirkung hintanhält. Bedingung für das Gelingen des Versuches ist es, dass die Wassermassen nicht zu gross gewählt werden und dass die Temperatur nicht allzu hoch sei, sonst kann es kommen, dass sogar bei von vornherein Kohlensäuregesättigten Wässern die Kohlensäuredurchleitung den Eisenausfall der Stehprobe gegenüber beschleunigt. Die Kohlensäurespannung ist im Wasser der Luft gegenüber sehr gross, unterstützt nun noch Bewegung im Wasser und hohe Temperatur die Abgabe, so wird bei grosser Wassermasse das Durchleiten der Kohlensäure diese Abgabe nicht compensiren können und gerade die durch die Gasblasen bewirkte Bewegung in der Flüssigkeit wird der ruhig stehenden Probe gegenüber eine Beschleunigung des Eisenausfalles bedingen. Ist die freiwillige Eisenausscheidung abhängig von dem Partialdruck, welcher im Wasser und in der über demselben stehenden Atmosphäre herrscht, so muss es möglich sein, durch Hinzugabe von Kohlensäure zum Stickstoff, ebenso wie zum Sauerstoff, oder zu einem Gemisch beider, also zu Luft, auf die Eisenausscheidung einzuwirken. Diesbezügliche Versuche wurden derart disponirt, dass jedes $\mathrm{MIal} 500^{\mathrm{ccm}}$ des eisenoxydulhaltigen Grundwassers unter 500 com einer verschieden zusammengesetzten Atmosphäre gestellt wurden, einer Atmosphäre, welche neben reinem Stickstoff oder Sauerstoff oder Luft $0 \cdot 1.3 \cdot 5 \cdot 7$ oder $\%$ Volumina reiner Kohlensäure enthielt. Die Wässer wurden in dem Augenblick benutzt, in welchem das Auftreten der ersten 
Spur jener initialen Opalescenz anzeigte, dass alle Bedingungen für die Eisenoxydation gegeben und diese eben eingeleitet sei. Zu diesem Zeitpunkte sind die Wässer für die Versuche deshalb am geeignetsten, weil sie bezüglich des Verhaltens des Eisens am gleichwerthigsten sind. Eisenbestimmungen liessen sodann beurtheilen, in welcher Weise die Natur des über dem Wasser stehenden Gasgemisches die Eisenoxydation beeinflusst hatte. Die folgenden Tabellen geben die Resultate.

a) Vergrösserung der $\mathrm{CO}_{2}$-Spannung im Stickstoff.

\begin{tabular}{|c|c|c|c|c|}
\hline $\begin{array}{c}\text { Menge des } \\
\text { Eisenwassers }\end{array}$ & $\begin{array}{l}\text { Zusammensetzung der } \\
\text { überstehenden Atmosphäre }\end{array}$ & $\begin{array}{l}\text { Eisengehalt } \\
\text { I. Versuch } \\
5 \text { Stunden }\end{array}$ & $\begin{array}{l}\text { I00 ccm nach } \\
\text { U. Versuch } \\
20 \text { Stunden }\end{array}$ & $\begin{array}{l}\text { Ablauf von } \\
\text { III. Versuch } \\
24 \text { Stunden }\end{array}$ \\
\hline $500 \mathrm{~cm}$ & $500 \mathrm{~cm} \mathrm{~N}$ & $1 \cdot 3^{\mathrm{mg}}$ & $1 \cdot 8^{\mathrm{mg}}$ & $1 \cdot 3^{\mathrm{mg}}$ \\
\hline ", & $450, ",+50 \mathrm{CO}_{2}$ & $1 \cdot 8$, & $2 \cdot 9$, & $1 \cdot 5$, \\
\hline , & $350, "+150$ & $2 \cdot 8$, & $3 \cdot \overrightarrow{5}$, & $3 \cdot 0$, \\
\hline , & $250,,+250$, & $2 \cdot 8$, & $3 \cdot \overline{5}$, & $3 \cdot 4$, \\
\hline , & $150, y+350$, & $2 \cdot 8$, & $3 \cdot \overline{5}$, & $3 \cdot 4$, \\
\hline " & $50,, \quad+450$, & $2 \cdot 8$, & $3 \cdot \overline{0}$, & $3 \cdot 4$, \\
\hline \multirow[t]{2}{*}{ ", } & $-\quad+500$ & $2 \cdot 8$, & $3 \cdot 5$, & $3 \cdot 4$, \\
\hline & $\begin{array}{l}\text { Eisengehalt des Grund. } \\
\text { wassers in } 100^{\mathrm{ccm}} \mathrm{Fe}=\end{array}$ & $2 \cdot 8$ & $3 \cdot \overline{0}$, & $3 \cdot 4$, \\
\hline
\end{tabular}

b) Vergrösserung der $\mathrm{CO}_{2}$-Spaninung im Sauerstoff.

\begin{tabular}{|c|c|c|c|c|}
\hline $\begin{array}{c}\text { Menge des } \\
\text { Eisenwassers }\end{array}$ & $\begin{array}{l}\text { Zusammensetzung der } \\
\text { überstehenden Atmosphäre }\end{array}$ & $\begin{array}{l}\text { Eisengehalt } \\
\text { I. Versuch } \\
7 \text { Standen }\end{array}$ & $\begin{array}{l}100^{\mathrm{ccm}} \text { nach } \\
\text { II. Versuch } \\
9 \text { Stunden }\end{array}$ & $\begin{array}{l}\text { Ablauf. von } \\
\text { III. Versuch } \\
24 \text { Stunden }\end{array}$ \\
\hline $500 \mathrm{cem}$ & $500 \mathrm{ecm} 0$ & $0.5 \mathrm{mg}$ & $0.2 \mathrm{mg}$ & $0.3^{\mathrm{mc}}$ \\
\hline " & $450,, \quad+\quad 50 \mathrm{CO}_{2}$ & $1 \cdot 5$, & 0.9, & $1 \cdot 0$, \\
\hline , & $350,, \quad+150$, & $2 \cdot 8$, & $3 \cdot 0$, & $1 \cdot 5$, \\
\hline , & $250,, \quad+250$ & $3 \cdot 3$, & $3 \cdot 4$, & $2 \cdot 4$, \\
\hline ,; & $150,,+350$, & $3 \cdot 3$, & $3 \cdot 4$, & $2 \cdot 8$, \\
\hline , & $50, "+450$ & $3 \cdot 3$, & $3 \cdot 4$, & $2 \cdot 8$, \\
\hline \multirow[t]{2}{*}{ " } & $-\quad+500$ & $3 \cdot 3$ & $3 \cdot 4$, & $2 \cdot 8$, \\
\hline & $\begin{array}{l}\text { Eisengehalt des Grund- } \\
\text { wassers in } 100^{\mathrm{cm}} \mathrm{Fe}=\end{array}$ & $3 \cdot 3$, & $3 \cdot 4$, & $2 \cdot 8$, \\
\hline
\end{tabular}


c) Vergrösserung der $\mathrm{CO}_{2}$-Spannung in Luft.

\begin{tabular}{|c|c|c|c|c|}
\hline $\begin{array}{c}\text { Menge des } \\
\text { Eisenwassers }\end{array}$ & $\begin{array}{l}\text { Zusammensetzung der } \\
\text { überstehenden Atmosphäre }\end{array}$ & $\begin{array}{l}\text { Eisengehalt } \\
\text { I. Versuch } \\
6 \text { Stunden }\end{array}$ & $\begin{array}{l}100 \mathrm{cmm} \text { nac } \\
\text { II. Versuch } \\
7 \text { Stunden }\end{array}$ & $\begin{array}{l}\text { Ablauf von } \\
\text { III. Versuch } \\
24 \text { Stunden }\end{array}$ \\
\hline $500 \mathrm{~cm}$ & $500^{\mathrm{com}} \mathrm{Luft}$ & $0.6 \mathrm{mg}$ & $0 \cdot 8^{\mathrm{mg}}$ & $0.4 \mathrm{mg}$ \\
\hline ", & $450,, \quad+50 \mathrm{CO}_{2}$ & $2 \cdot 1$, & $1 \cdot 7$, & $1 \cdot 2$, \\
\hline ", & $350, \quad,+150$, & $2 \cdot 4$, & $3 \cdot 0$, & $2 \cdot 4$, \\
\hline , & $250, \quad,+250$ & $2 \cdot 6$, & $3 \cdot 5$, & $2 \cdot 9$ \\
\hline , & $150,,+350$, & $2 \cdot 6$. & $3 \cdot 5$, & $2 \cdot 9$, \\
\hline , & $50,, \quad, 450$, & $2 \cdot 6$, & $3 \cdot 5$, & $2 \cdot 9$, \\
\hline \multirow[t]{2}{*}{$"$} & $-\quad+500$ & $2 \cdot 6$, & $3 \cdot 5$, & $2 \cdot 9$, \\
\hline & $\begin{array}{l}\text { Eisengehalt des Grund. } \\
\text { wassers in } 100 \mathrm{~cm} \mathrm{Fe}=\end{array}$ & $2 \cdot 6$, & $3 \cdot 5$, & $2 \cdot 9$, \\
\hline
\end{tabular}

Aus den vorstehenden Tabellen geht hervor, wie die Eisenausscheidung durch Erhöhung der Kohlensäurespannung beherrscht und verhindert werden kann und zwar dieses letztere sogar bei Gegenwart ron Sauerstoffmengen, welche an sich genügt hätten, um das Vielfache der Eisenmenge zu oxydiren, welche in den unterstehenden $500 \mathrm{~cm}$ Wasser enthalten waren. Wählt man das Wasserquantum kleiner oder grösser oder vermehrt oder vermindert man das Volumen der überstehenden Atmosphäre, so sieht man die Resultate sich verschieben, um sich immer wieder davon zu überzeugen, dass die Kohlensäure das Ausschlaggebende ist. Wir constatiren einen mächtigen, hemmenden Einfluss der Kohlensäure auf die Oxydation des Eisens, wenn wir sehen, dass nach wenigen Stunden (Versuch b.I) unter einer Atmosphäre reinen Sauerstoffes 84 Proc. des gesammten Eisens ausgefällt war, während unter einem Gemische von 90 Proc. Sanerstoff und 10 Proc. Kohlensäure in derselben Zeit nur 54 Proc. des Eisens oxydirt wurden. Bei 70 Proc. Sauerstoff +30 Proc. Kohlensäure sank die Ausscheidung auf 15 Proc., unter gleichen Mengen beider Gase aber auf Null.

Noch günstiger stellen sich die Verhältnisse, wenn neben der Kohlensäure nicht reiner Wasserstoff, sondern Luft zur Verwendung kam. Unter reiner Luft fielen hier (Versuch I c) 76 Proc. des Gesammteisens aus. 10 Proc. Kohlensäure drückten die Füllung auf 19 Proc. herab, während bei 30 Proc. nur noch 7 Proc., bei 50 Proc. aber nichts mehr oxydirt wurde. Unter der Stickstoffatmosphäre aber, bei welcher nur der im Wasser vorhandene Sauerstoff zur Wirkung kam, war schon bei 30 Proc. 
Kohlensäure keine Oxydation mehr zu beobachten, obwohl noch genügend freier Sauerstoff vorhanden war. Und zu alledem sind die die Oxydation aufhebenden Kohlensäurewerthe durch die Versuchsbedingungen im Sinne der Erhöhung beeinflusst. Genau $1000^{\mathrm{cem}}$ Inhalt messende Cylinder waren sorgfältig und ohne Luftblasen abzufangen, mit dem Eisenwasser gefüllt worden, um nunmehr mit Hülfe zweier Hahnauslässe ein entsprechendes Volumen Wasser durch Kohlensäure, ein weiteres durch Sauerstoff, Stickstoff oder Luft verdrängen zu lassen. Waren nun die Wässer immer erst in dem Augenblick in den Versuch gezogen, in welchem der Eisenausfall schon begann, so konnte naturgemäss diese Oxydation durch den im Wasser vorhandenen Sauerstoff so lange ungehindert fortschreiten, bis die über das Wasser gestellte Kohlensäure absorbirt wurde und wirken konnte. Nur dann wurde die Eisenausscheidung sofort sistirt, wenn relativ grosse Mengen von Kohlensäuremolecülen auf das Wasser trafen und somit eine ausgiebigere Aufnahme des Gases bewirkt wurde. Auch sieht man Luft und namentlich reinen Sauerstoff schon wirken, wenn sie durch die Flüssigkeit aufsteigen, man sieht sie also oxydiren, ohne dass die schon über der Flüssigkeit stehende Kohlensäure dies hindern kann. In den vorstehenden Versuchen wurde nun gezeigt, wie man den Eisenausfall durch Erhöhung der Kohlensäurespannung in der Atmosphäre gradatim verlangsamen kann; umgekehrt muss es möglich sein, durch Verminderung der Kohlensäurespannung eine Beschleunigung der Oxydation herbeizuführen. Dass man durch Verminderung der Kohlensäurespannung den Sauerstoff des Wassers zur Wirkung bringen kann, ist schon oben gezeigt worden, es soll nunmehr aber auch der Beweis erbracht werden, dass der Luftsauerstoff um so schneller wirkt, je mehr für die Bindung der vorhandenen Kohlensäure gesorgt wird.

Wir stellen einen Versuch an:

Von zwei grossen Krystallisationsschalen wird die eine mit einer Schicht ron starker Kalilauge, die andere mit gleich viel concentrirter Kochsalzlösung beschickt, um nunmehr zwei kleinere, $200 \mathrm{~cm}$ Eisenwasser enthaltende Schalen mitten hineinzustellen. Möglichst flache Glocken, welche eben in die grossen Schalen hineinpassen, und die zum Ausgleich des Luftdrucks vortheilhaft Hahnauslässe haben, sperren nun über dem Eisenwasser gleiche Luftrolumina ab. Die Kalilauge wird nun für eine rasche Bindung der Kohlensäure sorgen, während die concentrirte Kochsalzlösung ein indifferentes Sperrwasser abgeben wird. Die folgende Tabelle zeigt nun, wie die Erniedrigung der Kohlensäurespannung durch die Kalilauge den Eisenausfall beschleunigt. 


\begin{tabular}{|c|c|c|c|}
\hline & \multicolumn{3}{|c|}{$\begin{array}{l}\text { Eisengehalt in } 200 \mathrm{~cm} \text { nach Ablauf von } \\
\text { I. Versuch } \\
\begin{array}{c|c|c}\text { II. Versuch } & \text { III. Versuch } \\
\text { Stunde } & 4 \text { Stunden } & 8 \text { Standen }\end{array}\end{array}$} \\
\hline Kalilaugen-Abschluss & $2 \cdot 5^{\mathrm{mg}}$ & $0 \cdot 6^{\mathrm{mg}}$ & $1 \cdot 3 \mathrm{mg}$ \\
\hline Kochsalzlösung-Abschluss . . & $3 \cdot 1$, & $2 \cdot 0$, & $3 \cdot 2$, \\
\hline Ursprüngl. Eisengehalt des Grundwassers & $5 \cdot 2$, & $3 \cdot 1$, & $6 \cdot 0$, \\
\hline
\end{tabular}

Bedingung für das Gelingen des Versuches ist möglichst grosse Oberfläche und geringe Tiefe der Wasserschichten. Man könnte nun meinen, denselben Beweis auch damit liefern zu können, dass man mit Hülfe einer Luftpumpe eine Druckverminderung über dem Wasser erhält. Aber nur bei Wässern, welche reich an Sauerstoff, relativ arm aber an Kohlensäure sind, wird sich ein zeitlicher Tnterschied in der Eisenausscheidung dem Controlwasser gegenüber bemerkbar machen. Es ist ja auch zu bedenken, dass man beim Evacuiren nicht nur die Kohlensäure, sondern auch den Sauerstoff entfernt, und erklärt es sich mit diesen Thatsachen, dass man manchmal Eisenwässer unter einem luftverdünnten Raume überhaupt klar bleiben sieht. Bringt man aber ein mit Hülfe eines Vacuums gasfrei gemachtes Eisenwasser mit Luft ausgiebig in Berührung, so fällt naturgemäss das Eisen sehr schnell aus und wir sehen den Eisengehalt eines maschinell gehobenen Wassers energischer abnehmen, als den eines aus derselben Stelle geschöpften.

Auch aus diesen Erfahrungen geht zur Evidenz herror, dass die Entfernung der Kohlensäure aus dem Wasser für die Enteisenung von Bedeutung ist. Diese Ansicht wird überdies dadurch gestützt, dass man den praktischen Versuchen entsprechend ein sehr kohlensäurereiches Wasser zum Zwecke der Enteisenung intensiver durchlüften muss, als ein an jenem Gase armes, eine Thatsache, die auch darin ihren Ausdruck findet, dass sich ein Huminsubstanzen- und hiermit für gewöhnlich anch Kohlensäurereiches Wasser besser durch Berieselung als durch einfache Siebung enteisen lässt. ${ }^{1}$

Es liegt nun der Gedanke nahe, einem Wasser die Kohlensäure auch dadurch zu entziehen, dass man Kohlensäure bindende Körper in dasselbe einträgt. Sind diese Körper dem Eisenoxydul gegenüber an sich indifferent, so würde die von Fischer ${ }^{2}$ bestrittene Bedeutung der

1 Drei Berichte über die Wasserversorgung der Stadt Breslan mit Grundwasser von Hrn. Geheimen Medicinalrath Prof. Dr. Flügge in Breslau und Hrn. Baurath Thiem in Leipzig. Bericht III. S. 5.

2 Prof. Dr. Bernhard Fischer, Ueber das Grundwasser von Kiel mit besonderer Berücksichtigung seines Eisengehaltes nnd über Versuche zur Entfernung des Eisens aus demselben. Diese Zeitschrift. 1890. Bd. XIII. S. 304. 
Kohlensäure für die Eisenausscheidung eine weitere Stütze erhalten, wenn es sich zeigte, dass in den, mit jenen Kohlensäure bindenden Substanzen versetzten Wässern die Enteisenung eine beschleunigte ist. - Ein Versuch soll hierüber näheren Aufschluss geben.

Luftdicht verschliessbare Glaskolben werden rollständig bis zum Rande mit einem destillirten Wasser gefüllt, das eine bestimmte Menge Kohlensäure absorbirt enthielt, um nunmehr gleiche Volumina jener zu prüfenden Körper, welche nach Möglichkeit auf gleiche Korngrösse gebracht sind, einzutragen. Die Kolben werden schnell geschlossen und nach bestimmter Zeit auf ihren Gehalt an freier Kohlensäure geprüft. Für einen ersten Versuch zog ich frisch gefälltes Eisenoxydhydrat, geschlämmte Holzkohle, Feinsand und Cellulose in Form von Fliespapierbrei heran. Die folgende Tabelle zeigt, wie diese Substanzen, welche Sauerstoff und Kohlensäure frei eingebracht wurden, auf die absorbirte Kohlensäure des Kolbens wirkten:

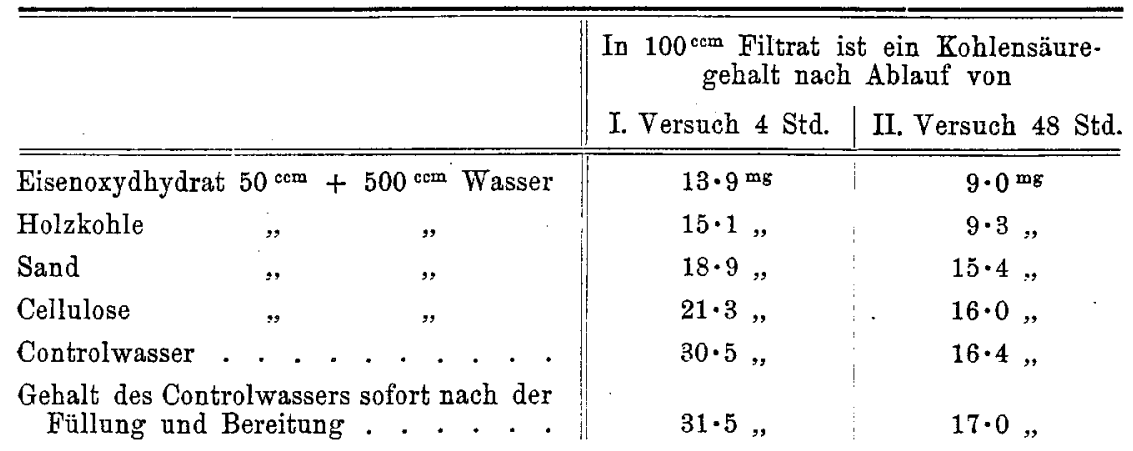

In einer zweiten Versuchsreihe wurde die Kohlensäure bindende Kraft der aufgeführten Körper volumenumetrisch geprüft.

$50 \mathrm{~cm}$ der Substanzen werden mit $500^{\mathrm{ccm}}$ destillirten Wassers in Kolben von $750 \mathrm{~cm}$ Inhalt gefüllt. Der Stopfen des Kolbens ist armirt mit einem Hahn sowie mit einem dünnen gebogenen Glasrohre, an welchem eine graduirte Bürette senkrecht herabhängt. Bürette und der über dem Wasser stehende Raum des Kolbens werden schnell mit Kohlensäure gefüllt, um jetzt die Büretten in eine Wanne mit concentrirter Kochsalzlösung ein Stück weit einzutauchen. Durch ein kurzes Oeffnen des am Kolbenverschluss befindlichen Hahnes wird Atmosphärendruck in den Büretten hergestellt und der Stand des Wassers schnell abgelesen. Wird nun die Kohlensäure in den verschiedenen Kolben in verschiedenem Grade gebunden, so muss dies in der Höhe der in den Büretten aufsteigenden Wassersäule zum Ausdruck kommen: 


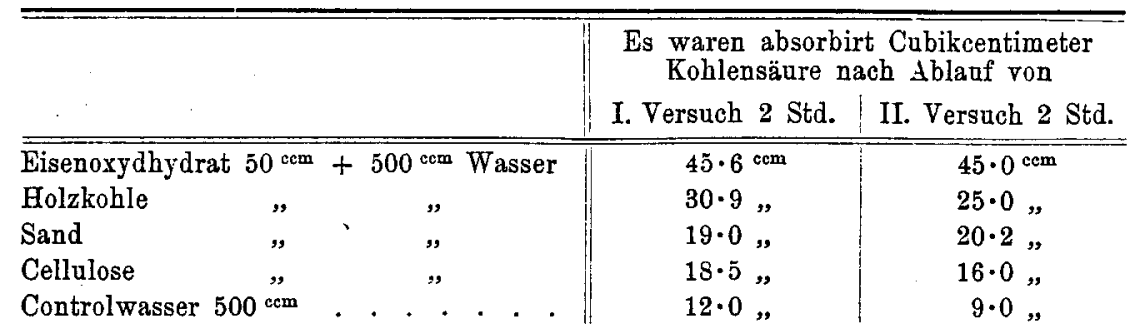

In sämmtlichen Versuchen, sowohl beim titrimetrischen als volumenmetrischen Bestimmungsmodus sehen wir nun ein bestimmtes Verhältniss der Kohlensäure bindenden Kräfte gewahrt. Am wirksamsten erwies sich jedes Mal das Eisenoxydhydrat, es folgt die Holzkohle, der Feinsand und schliesslich die Cellulose. Es erübrigt nunmehr darzuthun, wie sich jene Körper zum Ausfall des Eisens im Wasser verhalten. - Zu diesem Zweck versetze man in offen bleibenden Kolben $2000 \mathrm{~cm}$ eines Wassers von bestimmtem Eisengehalt mit je $50^{\mathrm{com}}$ der betreffenden Substanzen und prüfe nach dem Verlaufe von Stunden die Quantität des noch in Lösung befindlichen Eisenoxyduls.

Eisenfällung bei Gegenwart $\mathrm{CO}_{2}$ bindender Körper.

\begin{tabular}{|c|c|c|c|}
\hline I. Versuch & $\mid \begin{array}{c}\text { Eisengehalt } \\
1 \text { Stunde }\end{array}$ & $\begin{array}{l}\text { in } 100^{\mathrm{ccm}} \text { nach } \\
2 \text { Stunden }\end{array}$ & $\begin{array}{l}\text { Ablauf von } \\
3 \text { Stunden }\end{array}$ \\
\hline $\begin{array}{c}\text { In offenem Kolben Eisenoxydhydrat } \\
50^{\mathrm{ccm}}+2000^{\mathrm{cm}} \text { Wasser mit } 3 \cdot 2^{\mathrm{mg}} \\
\text { Eisen in } 100^{\mathrm{ccm}}\end{array}$ & 0 & 0 & 0 \\
\hline desgl. Holzkohle . . . . . . & $0 \cdot \overline{0}^{\mathrm{mg}}$ & $0 \cdot 1 \mathrm{mg}$ & 0 \\
\hline desgl. Sand . . & $1 \cdot 4$, & $1 \cdot 1$, & $0 \cdot 7 \mathrm{mg}$ \\
\hline 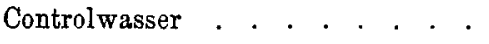 & $2 \cdot 5$, & $1 \cdot 7$, & $1 \cdot 2$, \\
\hline
\end{tabular}

Für diesen Versuch, welcher zeigt, wie der Eisenausfall beschleunigt wird, entsprechend den Kohlensäure bindenden Kräften der eingetragenen Körper, waren diese Substanzen von Sauerstoff und Kohlensäure befreit zur Verwendung gekommen. - Ist nun die Oxydation des Eisenoxyduls abhängig ron dem Kohlensäuregehalt des Wassers, ist es ferner richtig, dass jene Körper den Ausfall nur dadurch beschleunigten, dass sie Kohlensäure banden, so muss es möglich sein, das Eisenoxydhydrat, die Holzkohle, ebenso wie den Sand in ihrer Wirkung abzustumpfen, wenn sie schon mit Kohlensäure beladen in die Eisenwässer eingebracht werden. Wir entgasen daher für einen weiteren Versuch durch Erhitzen und lassen in reiner Kohlensäure erkalten. Für einen dritten Versuch schliesslich sättigen wir alle drei Körper bei $15^{\circ} \mathrm{C}$. mit Kohlensäure. 


\begin{tabular}{|c|c|c|c|}
\hline II. Versuch & \multicolumn{3}{|c|}{$\begin{array}{l}\text { Eisengehalt in } 100 \mathrm{~cm} \text { nach Ablauf von } \\
1 \text { Stunde } \mid 2 \text { Stunden } \mid 3 \text { Stunden }\end{array}$} \\
\hline $\begin{array}{l}\text { In offenem Kolben } 50^{\mathrm{ccm}} \text { Eisenoxydhydrat } \\
\text { erhitzt und unter } \mathrm{CO}_{2} \text { erkaltet }+2000^{\mathrm{ccm}} \\
\text { Wasser mit } 3 \cdot 1^{\mathrm{rgg}} \text { Eisen in } 100^{\mathrm{ccm}}\end{array}$ & $0.9 \mathrm{mg}$ & $0 \cdot 6^{\mathrm{mg}}$ & $0 \cdot 4^{\mathrm{mg}}$ \\
\hline desgl. Holzkohle . & $1 \cdot 9$ & $1 \cdot 5$, & 0.6, \\
\hline desgl. Sand . . . . . . & $2 \cdot 3$, & $2 \cdot 2$, & $1 \cdot 7$, \\
\hline Controlwasser & $2 \cdot 7$, & $2 \cdot 4$, & $2 \cdot 3$, \\
\hline
\end{tabular}

\begin{tabular}{|c|c|c|c|}
\hline III. $\mathrm{Versuch}$ & \multicolumn{3}{|c|}{ Eisengehalt in $100^{\mathrm{ccm}}$ nach Ablauf von } \\
\hline $\begin{array}{l}\text { In offenem Kolben } 50^{\mathrm{ccm}} \text { Eisenoxydhydrat } \\
\text { für } 15^{\circ} \text { C. mit } \mathrm{CO}_{2} \text { gesättigt }+2000^{\mathrm{ccm}} \\
\text { Wasser mit } 3 \cdot 2^{\text {nag }} \text { Eisen in } 100^{\text {com }}\end{array}$ & $1 \cdot 2^{\mathrm{mg}}$ & $1 \cdot 0^{\mathrm{mg}}$ & $0.7 \mathrm{mg}$ \\
\hline desgl. Holzkohle . . . . . & $2 \cdot 0$, & $1 \cdot 5$, & 0.8, \\
\hline desgl. Sand. . . & $2 \cdot 5$, & $2 \cdot 5$, & $1 \cdot 2$, \\
\hline Controlwasser & $2 \cdot 7$, & $2 \cdot 7$, & $2 \cdot 2$, \\
\hline
\end{tabular}

Hält man alle drei Versuche zusammen, so ergiebt sich wiederum ein deutlicher Einfluss der Kohlensäure auf die Eisenausscheidung. Die bekannte Thatsache, dass die Zugabe von Eisenoxydhydrat zu einem eisenhaltigen Wasser die Enteisenung beschleunigt, möchte ich einfach mit der Kohlensäure bindenden Kraft genannten Körpers erklären. Piefke ${ }^{1}$ deutet die Wirkung des Ferrihydrates damit, dass es bei Berührung mit oxydirbaren Körpern etwas Sauerstoff an dieselben abzugeben vermag. Es ist mir nicht gelungen, hierfür einen Beweis zu erbringen, da alle diesbezüglichen Versuche mit Eisenoxydhydrat höchstens eine Verdichtung unter Wasserabspaltung, aber keine Sauerstoffabgabe bewirken konnten. Mit dieser Kohlensäurebindung durch das Ferrihydrat erklärt es sich auch, dass das Verschwinden der freien Kohlensāure aus dem Eisenwasser gar nicht proportional dem Eisenausfall vor sich zu gehen braucht, ein Vorkommen, das Fischer gegen die Bedeutung der Kohlensäure für die Eisenausscheidung anführt. Dass die Kohlensäureabgabe in der ersten Zeit schneller vor sich geht als weiterhin, erklärt sich mit dem hohen Partialdruck, den die Kohlensäure im Wasser der Atmosphäre gegenüber besitzt. Dementsprechend ist auch in der ersten Zeit die Eisenausscheidung

${ }^{1}$ C. Piefke, Deber die Nutzbarmachang eisenhaltigen Grundwassers für die Wasserversorgung von Städten. Journal für Gasbeleuchtung und Wasserversorgung. 1891. XXXIV. Jahrg. Hft. 4 u. 5.

2 Fischer, a. a. 0 . 
eine ergiebigere. Mit der Annäherung an einen Spannungsausgleich aber nimmt die Schnelligkeit des Entweichens der Kohlensäure und hiermit auch die der Eisenausscheidung ab. Findet man nun von der Anfangs bestinmmten Menge eine Abnahme:

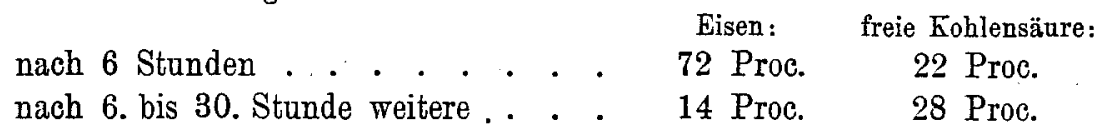

so erklärt sich dieses nicht Schritt halten mit der Thatsache, dass das schon ausgefallene Eisenoxydhydrat Kohlensäure bindet, das Wasser also Kohlensäure ärmer macht als es dem Eisenausfall entsprechend zu sein brauchte. So wird es auch nicht möglich sein, zu erweisen, dass für

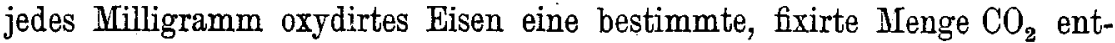
weicht. An dieser Stelle sei auch nachgetragen, dass jene Versuche, in welchen die Kohlensäurespannung im Stickstoff, im Sauerstoff und in der Luft durch ihre Erhöhung den Eisenausfall verlangsamte, absichtlich in ihren Resultaten nur eine Beobachtungsdauer bis zu 24 Stunden verzeichneten. Lässt man die Cylinder längere Zeit stehen, so beobachtet man, dass dann allmählich auch in den Gläsern Eisenausfällung eintrat, welche nach 24 Stunden noch keine Veränderung zeigten. Wenn nun oben mitgetheilt wurde, dass die Wässer erst dann in den Versuch gezogen wurden, wenn der Ausfall schon. begonnen hatte, so wissen wir nunmehr den Grund jener fortschreitenden Veränderung: Jenes schon von Anfang in Spuren vorhandene Eisenoxydhydrat bindet Kohlensäure und erniedrigt auf diese Weise mehr und mehr die Spannung dieses Gases, wodurch ein langsam fortschreitender Ausfall herbeigeführt wurde. Will man diese Eisenoxydhydratwirkung ausschalten und Resultate erhalten, welche von 24 Stunden ab unverändert bestehen bleiben, so nimmt man am besten geringe Nengen Wasser und sehr grosse Volumina der Atmosphären, am besten im Verhältniss von 1:8 bis 10; auch schöpft man das Wasser unter allen Cautelen in Cylindern und stülpt auf diese ebensolche mit der betreffenden Atmosphäre beschickte Gläser, so dass diese Gefässe durch ihre abgeschliffenen breiten Ränder auf einander gasdicht schliessen. Auch die Temperatur muss berücksichtigt werden, wenn man überlegt, dass die Absorptionsgrösse mit der Erhöhung der Temperatur abnimmt, so dass man am besten bei Temperaturen ron 8 bis $10^{\circ}$ arbeiten wird. Es entspricht dies am meisten den natürlichen Verhältnissen und kommt man so dem Verhalten von Bodenluft zum Grundwasser am nächsten, wobei die Ansicht vertreten werden soll, dass die Bodenluft unmittelbar über dem Grundwasser viel kohlensäurereicher ist, als man bisher glaubte. An einem anderen Orte soll diese Frage Würdigung finden. Für die vorliegenden Verhältnisse kommt sie weniger in 
Betracht, weil die Kohlensäure des Grundwassers rorwiegend anderer Provenienz ist.

Um nun auf jene mit Holzkohle, Sand u. s. w. angestellten Versuche zurückzukommen, so ergeben dieselben, däss sich die verwandten Substanzen kaum eignen werden, das Wasser eines Brunnens etwa dadurch eisenfrei machen zu wollen, dass man den Brunnenkessel mit jenen Körpern umgiebt und das Wasser zum Durchtritt durch dieselben zwingt. Es würde bald eine Ueberladung mit Kohlensäure und Wirkungslosigkeit eintreten. Für den Sand liegt dies ja der täglichen Erfahrung entsprechend auf der Hand. Es möchte daher eine Substanz gefunden werden, welche entsprechend grosse Mengen Kohlensäure zu binden vermag, ohne sich selbst schnell -zu verbrauchen, und dies Alles ohne das Auftreten von Umsetzungsproducten, welche die Nutzbarkeit des Wassers beeinträchtigen. - Man könnte an die Kreide denken. Wenn die Kohlensäure des Wassers Bicarbonat in Lösung bringt, fällt das Eisen aus und das Wasser würde nur eine geringe Vermehrung der Härte erfahren haben. Meine diesbezüglichen Untersuchungen haben ergeben, dass durch Hinzugabe ron gasfreier Kreide zu Eisenwasser eine Beschleunigung des Ausfalles erzielt wird. Wenn sogar in einem Kolben, der an einen, Kohlensäure entwickelnden Kipp'schen Apparate angeschlossen ist, unter dem Einfluss der Kreide Eisenausscheidung stattfindet, so beweist dies, dass die Kreide direct auf das Eisenbicarbonat wirkt und ihm ein Molecül Kohlensäure entzieht. So interessant diese Thatsachen auch sind, sie lassen sich für die Praxis nicht verwerthen, da die Wirkung eine viel zu langsame ist. Mit Hülfe von Chemicalien aber allein dürfte es möglich sein, auch für den Kleinbetrieb ohne maschinelle Hülfe ein eisenhaltiges Grundwasser nutzbar zu machen, ein Ziel, dessen Bedeutung ohne Weiteres auf der Hand liegt, wenn man überlegt, dass gar viele Orte, welche die bisher bekannten Enteisenungsverfahren der „Durchlüftung“ nicht einführen können, gezwungen sind, auf die einzige ideale Wasserversorgung durch Grundwasser zu verzichten. Eine in einem überwölbten Kesselbrunnen bewirkte Enteisenung aber hat überdies vor den Lüftungsverfahren immer noch den Vortheil voraus, dass jede Manipulirung mit dem Wasser vor dem Gebrauch vermieden ist, dass es, keimfrei wie es im Boden war, der Pumpenmündung entnommen werden kann.

Ein derartiges Verfahren besteht nach einem Herrn Baumeister Steckel zu Breslau ertheilten Patente in der Verwendung von Kalk. Aus porösen Ziegeln wird der Brunnen in zwei concentrischen Kreisen aufgemauert, so dass zwischen beiden Cylindermänteln ein Zwischenraum von etwa $10^{\circ \mathrm{m}}$ erhalten bleibt. Nunmehr wird Kalk gelöscht und, in dünner Schicht ausgebreitet, an der Luft liegend belassen. Ist der Kalk getrocknet, so bricht man ihn in 
Nussgrosse Stücke und fült mit diesen den Zwischenraum der Brunnenmäntel bis über das Niveau des höchsten Grundwasserstandes an. Auch die Brunnensohle wird etwa $10^{\mathrm{cm}}$ hoch mit den Kalkstücken bedeckt und über diese wiederum Sand geschichtet. Nunmehr kann das Pumpenrohr eingesetzt und der Kessel überwölbt werden. Ehe der Brunnen der Benutzung übergeben wird, lässt man ihn zweckmässig ein- oder zweimal am Tage eine Zeit lang hindurch auspumpen. Die Beobachtung dieser Brunnen ergab die folgenden Resultate: Der hohe Eisengehalt von 30 bis $40 \mathrm{mg}$ im Liter verschwand sofort, d. h. bald nach Fertigstellung des Brunnens war das dem Pumpenrohr entnommene Wasser eisenfrei und bewahrt der Brunnen diese Eligenschaft auch für die Dauer wie ein nunmehr 17 Jahre benutzter Brunnen beweist, eine Anlage, welche den Bedarf eines grossen Vergnügungsetablissements zu decken und einen Gemüsegarten zu versorgen hat. In den ersten Tagen enthielt das Wasser freies Alkali, welches aber um so eher verschwindet, je öfter in der ersten Zeit der Brunnen leergepumpt wurde. Für die Dauer aber ist die Härte des Wassers vermehrt, dies aber nicht derart, dass hierdurch eine Beanstandung zu begründen wäre. Pumpt man den Brunnen leer und entnimmt eine Probe des frisch in den Kessel eintretenden Wassers, so überzeugt man sich, dass auch dieses Wasser Eisen und vor allem Aetzalkali frei ist. Naschinell forcirt sind derartige Brunnen bisher nicht und fehlt daher die Erfahrung, von welcher Beschaffenheit das durch die Kalkschicht hindurchgerissene Wasser sein würde. Der Zufluss in einem derartigen Brunnenkessel ist einem gewöhnlichen Brunnen gegenüber naturgemäss etwas verlangsamt, aber bei weitem nicht so, dass dies gegen die Brauchbarkeit namentlich im Kleinbetriebe irgendwie sprechen könnte. Ich gebe im Folgenden Analysen des ursprünglichen Grundwassers und der frisch an der Sohle der ausgepumpten Brunnen entnommenen Proben.

I.

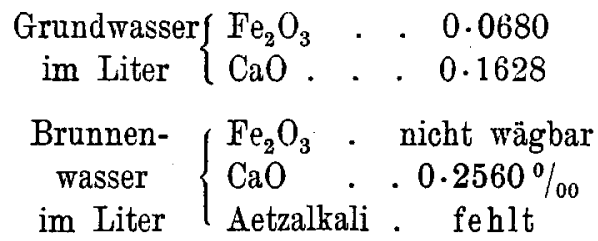

II.

$$
0 \cdot 0460
$$$$
0 \cdot 1320
$$$$
\begin{gathered}
\text { nicht wägbar } \\
0.2770^{\circ} \% \\
\text { fehlt }
\end{gathered}
$$

III. 0.0520 $0 \cdot 1470$

0.0006 $0.2600 \%$ fehlt.

Die Brauchbarkeit des Kalkes geht aus diesen Zahlen deutlich hervor, merkwürdig erscheint nur auf den ersten Blick das schnelle Verschwinden des Aetzkalkes aus dem Wasser, und besonders auch, dass dies geschieht unbeschadet der Wirkung des Brunnens auf das gelöste Eisen. Lässt man über ein Stück jenes Kallkhydrates ein stark eisenhaltiges Wasser fliessen, 
so bedeckt es sich sehr bald mit einer Schicht rothbraunen Ferrihydrates und unter dem Einfluss der Kohlensäure des Wassers verwandeln sich die oberflächlichen Schichten in Calciumcarbonat. Drei Körper sind es also, welche nunmehr auf das gelöste Eisen einwirken können: das Kalkhydrat, das Carbonat und das Eisenoxydhydrat. Reichliche Gelegenheit für die Bindung der das Fisen in Lösung haltenden Kohlensäure ist also gegeben, die geringe Menge Kalkhydrat aber, welche das Wasser aus dem Inneren der Stücke in Lösung bringt, beseitigt das Eisenoxydhydrat auf zwei Wegen: Wir sahen, welche grossen Massen Kohlensäure das Eisenoxydhydrat binden kann und diese Kohlensäure ist es, welche vorwiegend die Alcalescenz abstumpft. Diese auf dem Ferrihydrat verdichtete Kohlensäure fällt den Kalk als Carbonat und macht die Eisenoxydverbindung fähig, immer von Neuem wieder wirksame Kohlensäure aufzunehmen. Ein anderer Theil des gelösten Aetzkalkes geht direct mit dem Ferrihydrat eine feste Verbindung ein, aus der das Alkali durch Auswaschen nicht zu entfernen ist, eine Thatsache, welche in dem Verhalten des Ferrihydrates zu anderen Alkalien, z. B. Ammoniak, ihr dnalogon findet. Man nehme einmal frisch gefälltes Eisenoxydhydrat und gebe Kalkwasser nach und nach in kleinen Portionen hinzu, um sich zu überzeugen, wie rasch die Alkalescenz verschwindet und wie grosse Mengen Aetzkalk ein Kohlensäure beladenes Ferrihydrat zu fällen vermag. Die Zunahme der Härte des Wassers ist also nur durch jene geringe Nlengen Calciumbicarbenat bedingt, welche in Lösung gehen.

Auf Grund aller dieser Thatsachen empfiehlt sich das Steckel'sche Enteisenungsverfahren für Pumpbrunnen ganz besonders bei allen sehr stark eisenhaltigen Wässern und muss es freudig begrüsst werden, dass nunmehr auch der Kleinbetrieb über ein brauchbares Verfahren verfügt, welches die grosse Frage der Wasserversorgung lösen hilft. 